\section{PWE-004 SALAHADEEN (STUDY ASSESSING LANDMARK HEIGHT ALTERATION DURING ENDOSCOPIC EVALUATION)}

doi:10.1136/gut.2011.239301.267

N E Burch, ${ }^{1, *}$ O Saraj, ${ }^{2}$ B Lichfield, ${ }^{1}$ D Bools, ${ }^{1}$ J Jankowski ${ }^{1}$ Digestive Diseases Department, University Hospitals Leicester NHS Trust, Leicester, UK; ${ }^{2}$ Scarborough General Hospital, Scarborough, UK

Introduction Accurate identification and measurement of relevant landmarks at upper GI endoscopy is a basic and important aspect of endoscopic evaluation. The aim of this study was to evaluate whether there was any difference between landmark readings at upper GI endoscopy on intubation versus extubation, with a view to standardising how these measurements are taken.

Methods All patients undergoing diagnostic upper GI endoscopy between September 2009 and April 2010 within University Hospitals Leicester were identified for potential recruitment. Landmark measurements were recorded on both insertion and withdrawal of the endoscope. Measurements taken included: gastro-oesophageal junction (GOJ); diaphragm; hiatus hernia $(\mathrm{HH})$; and barretts length (C\&M classification). Measurements taken on withdrawal of the scope were recorded after the stomach was fully emptied of air by the endoscopist.

Results 259 patients were included in the trial. 3 endoscopists recruited patients, with the majority of data collected by a single endoscopist. Patient age ranged between 18 and 95 years. Hiatus Hernia: $74 \%$ were found to have a HH. In $2 \%$ there was a $\mathrm{HH}$ on intubation, but no $\mathrm{HH}$ on extubation. $23 \%$ had a $\mathrm{HH}$ on extubation, but no $\mathrm{HH}$ on intubation. $\mathrm{HH}$ size varied from $-2 \mathrm{~cm}$ to $+4 \mathrm{~cm}$. Mean HH size was larger when measured on extubation than intubation $(\mathrm{p}=0.032)$.

Barretts Oesophagus: Variation in length of Barretts from intubation to extubation was -2 to $+6 \mathrm{~cm}$, with the length of Barretts being significantly shorter when measured on extubation ( $\mathrm{p}=0.003)$.

Gastro-oesophageal Junction: Difference in GOJ location varied from -5 to $+4 \mathrm{~cm}$, with the GOJ being at a significantly more proximal measurement when measured on extubation $(\mathrm{p}<0.0001)$.

Diaphragm: There was no significant difference in diaphragmatic measurements taken on insertion of the scope versus scope withdrawal ( $p$ 0.201).

Conclusion Specific landmark measurements at upper GI endoscopy are significantly influenced by whether taken on insertion or withdrawal of the endoscope.

The more proximal measurement of the GOJ on withdrawal may reflect oesophageal shortening secondary to longitudinal muscle contraction resulting from presence of the endoscope within the oesophagus. A HH may also be pulled proximally via the same process, accounting for the larger $\mathrm{HH}$ when evaluated on withdrawal. Diaphragmatic indentation is unaffected by insertion/withdrawal since it is extrinsic to the GO tract. Length of Barretts was significantly shorter when evaluated on endoscope withdrawal. This may result in missed diagnosis of short-segment Barretts, impact upon CM classification, and may lead to inaccurate mapping of dysplasia. The authors advocate a standardised method for landmark measurements at upper GI endoscopy in order to combat some of these discrepancies.

Competing interests None.

Keywords Barrett's oesophagus, endoscopic classification, landmark measurement, oesophageal physiology. 